\title{
On local and global universal relations in elasticity
}

\author{
ALAN WINEMAN and MUKESH GANDHI \\ Department of Mechanical Engineering and Applied Mechanics, The University of Michigan, Ann Arbor, MI \\ 48109, USA
}

(Received March 29, 1982)

\section{Introduction}

In the nonlinear theory of isotropic elastic materials, there are relations between force-like quantities and deformation-like quantities which are independent of material properties. Such relations have been called "universal" relations [1]. The best known example is that of Rivlin for simple shear [2]. It relates components of normal and shear stress and the amount of shear. Another important example arises in the problem of the combined extension and torsion of a circular cylinder. Rivlin [3] showed that for small angles of twist superposed on finite extension, the ratio of the twisting moment to the angle of twist depends only on the axial force and the extension ratio. In this note relations of the first type, involving components of the stress and stretch tensors, will be referred to as "local". Relations of the second type, involving resultant forces or moments, will be referred to as "global".

A general method for constructing local universal relations has been presented by Hayes and Knops [4]. In section 2, this method is used to derive a universal relation for the homogenous deformation consisting of simple shear superposed on triaxial extension. This is the local deformation which each material element undergoes in the non-homogeneous deformation of combined inflation, extension and torsion of a circular cylinder. Rivlin's global universal relation for this problem is normally obtained by first deriving expressions for the resultant force and moment in terms of the strain energy density and stretch tensor components, and then comparing them. In section 3, it is shown that Rivlin's global universal relation can be derived from the local universal relation of section 2 . Finally, in section 4 , global universal relations are established for small amplitude axial or circumferential shearing superposed on finite extension and inflation of a circular cylinder.

\section{Local universal relation for shear superposed on triaxial extension}

Consider a body whose shape in the reference state is a cube. With respect to a cartesian coordinate system, let $X_{i}$ denote the coordinates of a particle in the reference state and $x_{i}$ denote its coordinates in a deformed state. Let the cube be subjected to the homogeneous deformation

$$
x_{1}=\lambda_{1} X_{1}+\kappa \lambda_{2} X_{2}, \quad x_{2}=\lambda_{2} X_{2}, \quad x_{3}=\lambda_{3} X_{3} \text {. }
$$

In this deformation the cube undergoes length changes along each coordinate axis, 
followed by a simple shear of planes $x_{2}=$ constant in the $x_{1}$ direction. Parameter $\kappa$ represents the displacement along the $x_{1}$ axis per unit current length in the $x_{2}$ direction. In the case of incompressible materials, which is all that is considered here, $\lambda_{1} \lambda_{2} \lambda_{3}=1$.

The deformation gradient will be denoted by $\boldsymbol{F}=\left[\partial x_{i} / \partial X_{j}\right]$ with respect to cartesian coordinates. The local universal relations derived by the method of Hayes and Knops are expressed in terms of the components of the Cauchy stress tensor and the components of the principal vectors of the Cauchy-Green tensor $\boldsymbol{B}=\boldsymbol{F F} \boldsymbol{F}^{T}$.

For the deformation in Eqn. (1) the principal values for $\boldsymbol{B}$ are found to be

$$
a_{1}=A+B, \quad a_{2}=A-B, \quad a_{3}=\lambda_{3}^{2},
$$

where

$$
\begin{aligned}
& A=\frac{1}{2}\left[\lambda_{1}^{2}+\lambda_{2}^{2}+\kappa^{2} \lambda_{2}^{2}\right] \\
& B=\frac{1}{2}\left[\left(\lambda_{1}^{2}+\lambda_{2}^{2}+\kappa^{2} \lambda_{2}^{2}\right)^{2}-4 \lambda_{1}^{2} \lambda_{2}^{2}\right]^{1 / 2}
\end{aligned}
$$

These are seen to be distinct. The principal vectors corresponding to $a_{1}, a_{2}, a_{3}$, denoted respectively by $\boldsymbol{R}, \boldsymbol{S}, \boldsymbol{T}$, are found to be

$$
\begin{aligned}
& \boldsymbol{R}=\frac{\left(A+B-\lambda_{2}^{2}\right) i+\kappa \lambda_{2}^{2} j}{\left[\kappa^{2} \lambda_{2}^{4}+\left(A+B-\lambda_{2}^{2}\right)^{2}\right]^{1 / 2}}, \\
& \boldsymbol{S}=\frac{\left(A-B-\lambda_{2}^{2}\right) i+\kappa \lambda_{2}^{2} j}{\left[\kappa^{2} \lambda_{2}^{4}+\left(A-B-\lambda_{2}^{2}\right)^{2}\right]^{1 / 2}}, \\
& \boldsymbol{T}=\boldsymbol{k},
\end{aligned}
$$

where $\boldsymbol{i}, \boldsymbol{j}, \boldsymbol{k}$ are the unit base vectors for the cartesian coordinate system.

According to the method of Hayes and Knops, since $a_{1}, a_{2}, a_{3}$ are distinct, there are three universal relations, given by

$$
\sigma_{i j} R_{i} S_{j}=0, \quad \sigma_{i j} R_{i} T_{j}=0, \quad \sigma_{i j} S_{i} T_{j}=0
$$

Using Eqns. (2) and (3), one finds that Eqns. (4) imply

$$
\sigma_{11}-\sigma_{22}=\left(\frac{\lambda_{1}^{2}+\lambda_{2}^{2} \kappa^{2}-\lambda_{2}^{2}}{\kappa \lambda_{2}^{2}}\right) \sigma_{12},
$$

together with $\sigma_{13}=\sigma_{23}=0$.

The local universal relation (5) has several interesting features. First, it has the same form as that for simple shear, $\left(\lambda_{1}=\lambda_{2}=\lambda_{3}=1\right)$, in which the parameters defining the deformation occur only in the coefficient of $\sigma_{12}$. Secondly, if $\lambda_{1}=\lambda_{2}$, i.e. if there is equal biaxial extension or contraction along the $X_{1}$ and $X_{2}$ axes, then (5) reduces to

$$
\sigma_{11}-\sigma_{22}=\kappa \sigma_{12} \text {. }
$$

This is the same form as for simple shear, and is independent of the length change of the edges of the cube. Finally, two special cases of the general relation are recorded: (a) for simple shear superposed on uniaxial extension along the $X_{1}$-axis, let $\lambda_{1}=\lambda$, $\lambda_{2}^{2}=\lambda_{3}^{2}=\lambda^{-1}$. Then,

$$
\sigma_{11}-\sigma_{22}=\left[\frac{\lambda^{3}+\kappa^{2}-1}{\kappa}\right] \sigma_{12},
$$


(b) for simple shear superposed on uniaxial extension along the $X_{2}$-axis, let $\lambda_{2}=\lambda$, $\lambda_{1}^{2}=\lambda_{3}^{2}=\lambda^{-1}$. Then

$$
\sigma_{11}-\sigma_{22}=\left(\frac{\lambda^{-3}+\kappa^{2}-1}{\kappa}\right) \sigma_{12} \text {. }
$$

\section{Rivlin's global universal relation}

Consider a hollow circular cylinder of an incompressible non-linear isotropic elastic material. In its reference state, its inner and outer radii are $R_{1}$ and $R_{2}$, respectively. The cylinder is to be subjected to a deformation consisting of combined inflation, extension and twisting. If the cylinder is coaxial with a circular cylindrical coordinate system, then a particle at $(R, \Theta, Z)$ in the reference state moves to coordinates $(r, \theta, z)$ according to the relation,

$$
r=r(R), \quad \theta=\Theta+\psi \lambda Z, \quad z=\lambda Z,
$$

where $\lambda$ and $\psi$ are constant, and $\psi$ represents the rotation of a plane cross-section per unit current length.

As a typical particle in the current state, the components of the deformation gradient tensor with respect to unit base vectors $\boldsymbol{e}_{r}, \boldsymbol{e}_{\theta}, \boldsymbol{e}_{z}$ are given by

$$
\boldsymbol{F}=\left[\begin{array}{lll}
\mathrm{d} r / \mathrm{d} R & 0 & 0 \\
0 & r / R & r \psi \lambda \\
0 & 0 & \lambda
\end{array}\right]
$$

Consider a local cartesian coordinate system with unit base vectors $e_{1}=e_{\theta}, e_{2}=e_{z}$, $e_{3}=e_{r}$. Then the deformation gradient in (10) is the same as that for (1), where $\lambda_{1}=r / R, \lambda_{2}=\lambda, \lambda_{3}=\mathrm{d} r / \mathrm{d} R$ and $\kappa=r \psi$. In other words, each particle of the cylinder undergoes a local homogeneous deformation consisting of extension or contraction in the local coordinate directions followed by simple shear. With respect to local cartesian coordinates, the components of stress and $\boldsymbol{F}$ must satisfy the universal relation (5). It follows that at each particle the stress components with respect to $e_{r}, e_{\theta}, e_{z}$ and components of $\boldsymbol{F}$ in (10) must satisfy

$$
\sigma_{\theta \theta}-\sigma_{z z}=\left[\frac{(r / R)^{2}+\lambda^{2}\left[(r \psi)^{2}-1\right]}{r \psi \lambda^{2}}\right] \sigma_{\theta z} .
$$

Now, consider the special case of the extension and twisting of a solid rod. In this case $R_{1}=r\left(R_{1}\right)=0$. It follows from the incompressibility constraint that

$$
r=R / \sqrt{\lambda} \text {. }
$$

Then Eqn. (11) can be rewritten as

$$
\sigma_{z \theta}=\left[\frac{\psi \lambda^{2} r}{\lambda^{2}-\lambda^{-1}-(\psi \lambda r)^{2}}\right]\left(\sigma_{z z}-\sigma_{\theta \theta}\right) .
$$

The twisting moment on the end of the rod is

$$
M=2 \pi \int_{0}^{r_{2}} r^{2} \sigma_{z \theta} \mathrm{d} r
$$


and the axial force is

$$
N=2 \pi \int_{0}^{r_{2}} \sigma_{z z} r \mathrm{~d} r,
$$

where $r_{2}=r\left(R^{2}\right)$ is the radius of the deformed rod. It follows from relation (13) that

$$
M / \psi=2 \pi \lambda^{2} \int_{0}^{r_{2}} \frac{r^{3}\left(\sigma_{z z}-\sigma_{\theta \theta}\right)}{\lambda^{2}-\lambda^{-1}-(\psi \lambda r)^{2}} \mathrm{~d} r .
$$

It is shown in [3] that $\sigma_{\theta \theta}=\mathrm{O}\left(\psi^{2}\right)$ and $\sigma_{z z}=\sigma_{0}(\lambda)+\mathrm{O}\left(\psi^{2}\right)$, where $\sigma_{0}(\lambda)$ is the spatially constant stress on a cross-section of the rod in uniaxial extension. Then from Eqn. (16)

$$
M /\left.\psi\right|_{\psi=0}=\frac{\lambda^{2}}{\lambda^{2}-\lambda^{-1}} \frac{1}{2} \pi r_{2}^{4} \sigma_{0}(\lambda),
$$

and from Eqn. (15)

$$
\left.N\right|_{\psi=0}=\sigma_{0}(\lambda) \pi r_{2}^{2} .
$$

Finally, it follows from Eqns. (12), (17) and (18) that

$$
\left(\frac{N}{M / \psi}\right)_{\psi=0}=\frac{2}{R_{0}^{2}}\left(\lambda-\frac{1}{\lambda^{2}}\right),
$$

which is Rivlin's global universal relation. Thus, it is seen that this relation is a consequence of the fact that the same general form of local universal relation holds at each material particle, the amount of shear $\kappa(r)=\psi r$ varying with particle radius.

\section{Extension, inflation and shear of a cylinder}

Consider the same cylinder as in section 3, but which is now subjected to extension, inflation and circumferential shear about its axis. This is a special case of the general shear problem discussed by Rivlin [3]. A particle at $(R, \Theta, Z)$ in the reference state moves to coordinates $(r, \theta, z)$ according to the relation

$$
\begin{aligned}
& r=r(R), \\
& \theta=\Theta+\phi(R), \\
& z=\lambda Z .
\end{aligned}
$$

With respect to unit base vectors $\boldsymbol{e}_{r}, \boldsymbol{e}_{\theta}, \boldsymbol{e}_{z}$, the components of the deformation gradient are given by

$$
\boldsymbol{F}=\left[\begin{array}{lll}
r^{\prime} & 0 & 0 \\
r \boldsymbol{\phi}^{\prime} & r / R & 0 \\
0 & 0 & \lambda
\end{array}\right],
$$

where ( $)^{\prime}=\mathrm{d}() / \mathrm{d} R$.

Consider a local cartesian coordiante system with base vectors $\boldsymbol{e}_{1}=\boldsymbol{e}_{\boldsymbol{\theta}}, \boldsymbol{e}_{2}=\boldsymbol{e}_{r}$, $e_{3}=-e_{z}$. Then the deformation gradient given by (21) is the same as that for a local homogeneous deformation of the form given by Eqn. (1) with $\lambda_{1}=r / R, \lambda_{2}=r^{\prime}$, $\lambda_{3}=\lambda, \kappa=r \phi^{\prime} / r^{\prime}, \sigma_{11}=\sigma_{\theta \theta}, \sigma_{22}=\sigma_{r r}$ and $\sigma_{12}=\sigma_{r \theta}$. For notational convenience, let 
$r / R=\mu$. The incompressibility constraint requires that $r^{\prime}=(\lambda \mu)^{-1}$. It then follows from the discussion in section 3 and relation (5) that at each point in the cylinder, there is satisfied the relation

$$
\sigma_{\theta \theta}-\sigma_{r r}=\frac{(\lambda \mu)\left[\mu^{2}-(\lambda \mu)^{-2}+\left(r \phi^{\prime}\right)^{2}\right]}{r \phi^{\prime}} \sigma_{r \theta} .
$$

Both $\mu$ and $\phi$ are functions of $R$. The specific form of $\mu$ is determined by the incompressibility condition, and does not depend on the form of the strain energy function. On the other hand, as shown in [3], $\phi(R)$ satisfies a nonlinear ordinary differential equation whose form is determined by the choice of the strain energy function. Thus, the local relation (22) does not appear to be very useful. However, a universal relation among externally measured quantities can be constructed from relation (22) under certain conditions.

The inner and outer radii in the deformed configuration are denoted by $r_{1}=r\left(R_{1}\right)$ and $r_{2}=r\left(R_{2}\right)$, respectively. The shearing moments on the inner and outer surfaces must have the same magnitude $M$ given by

$$
M=2 \pi\left(\lambda L_{0}\right) r_{1}^{2} \sigma_{r \theta}\left(R_{1}\right)=2 \pi\left(\lambda L_{0}\right) r_{2}^{2} \sigma_{r \theta}\left(R_{2}\right),
$$

where $L_{0}$ is the unextended length of the cylinder. On the inner and outer surface, let

$$
\sigma_{r r}\left(R_{1}\right)=-p_{1}, \quad \sigma_{r r}\left(R_{2}\right)=-p_{2} .
$$

We restrict attention to small circumferential shear of the cylinder superposed on finite extension and inflation. Then, neglecting terms of $\mathrm{O}\left(\phi^{\prime 2}\right)$, Eqn. (22) reduces to

$$
\sigma_{\theta \theta}-\sigma_{r r}=\lambda \mu\left[\mu^{2}-(\lambda \mu)^{-2}\right] \frac{\sigma_{r \theta}}{\left(r \phi^{\prime}\right)},
$$

in which $\sigma_{\theta \theta}$ and $\sigma_{r r}$ are now regarded as stresses associated with the problem of finite extension and inflation of the cylinder. Let it be further assumed that the cylinder is thin walled, i.e. $\left(R_{2}-R_{1}\right) / R_{1} \ll 1$. It follows from the equilibrium equations that

$$
\sigma_{\theta \theta}\left(R_{2}\right)=\frac{p_{1} r_{1}-p_{2} r_{2}}{r_{2}-r_{1}}+\mathrm{O}\left(R_{2}-R_{1}\right) .
$$

Furthermore, we introduce the approximation

$$
\begin{aligned}
& \phi^{\prime}\left(R_{2}\right)=\frac{\phi_{2}-\phi_{1}}{R_{2}-R_{1}}+\mathrm{O}\left(R_{2}-R_{1}\right), \\
& \mu=\mu(R)=\mu\left(R_{2}\right)+\mathrm{O}\left(R_{2}-R_{1}\right),
\end{aligned}
$$

where $\phi_{i}=\phi\left(R_{i}\right)$. On evaluating relation (25) at $r=r_{2}$, then combining Eqns. (23)-(27), and simplifying, one obtains the following relation to within an error of $\mathrm{O}\left(R_{2}-R_{1}\right)$

$$
\frac{M}{\phi_{2}-\phi_{1}}=\frac{2 \pi L_{0} R_{2}^{4}}{\left(R_{2}-R_{1}\right)^{2}}\left(p_{1}-p_{2}\right) \frac{\mu^{4}}{\mu^{2}-(\lambda \mu)^{-2}} \text {. }
$$

This is a universal relation which gives the small amplitude circumferential shearing stiffness in terms of the pressure difference, geometric properties of the undeformed cylinder, and then axial and circumferential stretch ratios of the outer surface.

In a similar manner, a global universal relation can be established for the axial 
shearing stiffness of the cylinder. In this case, the cylinder is subjected to the deformation

$$
r=r(R), \quad \theta=\Theta, \quad z=\lambda Z+w(R) .
$$

With respect to base vectors $\boldsymbol{e}_{r}, \boldsymbol{e}_{\theta}, \boldsymbol{e}_{z}$, the components of the deformation gradient are

$$
\boldsymbol{F}=\left[\begin{array}{lll}
r^{\prime} & 0 & 0 \\
0 & r / R & 0 \\
w^{\prime} & 0 & \lambda
\end{array}\right]
$$

This is the same form of deformation gradient as for a local homogeneous deformation of form (1) if $e_{1}=e_{z}, e_{2}=e_{r}$, and $e_{3}=e_{\theta}$. Then $\lambda_{1}=\lambda, \lambda_{2}=r^{\prime}, \lambda_{3}=r / R, \kappa=w^{\prime} / r^{\prime}$, $\sigma_{11}=\sigma_{z z}, \sigma_{22}=\sigma_{r r}$ and $\sigma_{12}=\sigma_{r z}$. It follows from relation (5) that at each point,

$$
\sigma_{z z}-\sigma_{r r}=(\lambda \mu) \frac{\left[\lambda^{2}-(\lambda \mu)^{-2}+\left(w^{\prime}\right)^{2}\right]}{w^{\prime}} \sigma_{z r} \text {. }
$$

The shearing forces on the inner and outer cylindrical surfaces have the same magnitude $T$ given by

$$
T=2 \pi\left(\lambda L_{0}\right) r_{2} \sigma_{z r}\left(R_{2}\right)=2 \pi\left(\lambda L_{0}\right) r_{1} \sigma_{z r}\left(R_{1}\right) .
$$

If relation (31) is evaluated at $r_{1}$ or $r_{2}$, then $\sigma_{r r}$ and $\sigma_{z r}$ can be expressed in terms of externally measured variables using relations (24) and (32). In order to do the same for the other variables, attention is now restricted to the case of small amplitude axial shear superposed on finite extension and inflation of a thin walled cylinder. Then terms of $\mathrm{O}\left(w^{\prime 2}\right)$ can be neglected in relation (31). To within term of $\mathrm{O}\left(R_{2}-R_{1}\right)$

$$
\begin{aligned}
& w^{\prime}(R)=\frac{w_{2}-w_{1}}{R_{2}-R_{1}}, \\
& \sigma_{z z}(R)=\sigma_{z z}\left(R_{2}\right), \quad \mu(R)=\mu\left(R_{2}\right),
\end{aligned}
$$

where $w_{i}=w\left(R_{i}\right)$. The axial force $N$ is given by

$$
N=\sigma_{z z} \pi\left(r_{2}^{2}-r_{1}^{2}\right)=\frac{\sigma_{z z} \pi}{\lambda}\left(R_{2}^{2}-R_{1}^{2}\right),
$$

where use has been made of the result due to incompressibility, that

$$
r^{2}=R^{2} / \lambda+C
$$

where $C$ is some constant. Finally, evaluating (31) at $r_{2}$, combining (32)-(34) and simplifying, we obtain the desired result

$$
\frac{T}{w_{2}-w_{1}}=\left[\frac{\lambda N}{\pi\left(R_{2}^{2}-R_{1}^{2}\right)}+p_{2}\right] \frac{R_{2}}{R_{2}-R_{1}} 2 \pi L_{0}\left(\lambda^{2}-\frac{1}{(\lambda \mu)^{2}}\right)^{-1} \text {. }
$$

\section{References}

[1] C. Truesdell and W. Noll, The Non-Linear Fleld Theories of Mechanics, Handbuch der Physik III/3 (1965).

[2] R.S. Rivlin, Some Applications of Elasticity Theory to Rubber Engineering, Proc. 2nd. Tech. Conf. (London, June 23-25, 1948), Cambridge: Heffer.

[3] R.S. Rivlin, Large Elastic Deformations of Isotropic Materials, VI. Further results in the Theory of Torsion, Shear and Flexure," Phil. Trans. Royal. Soc., London, A242, (30 December, 1949), pp. 173-195.

[4] M. Hayes and R.J. Knops, On universal relations in elasticity theory, ZAMP 17 (1966) 636-639. 